\title{
Clinical Characteristic of Thoracic Malignancy Patients with Coronavirus Disease 2019 Related to Mitigation Strategy in Dharmais National Cancer Center Hospital, Indonesia
}

\author{
Arif Hanafi ${ }^{1 *}\left(\mathbb{D}\right.$, Noorwati Soetandyo ${ }^{2}$, Achmad Mulawarman Jayusman ${ }^{1}$, Leovinna Widjaja ${ }^{3}$, Fifi Dwijayanti ${ }^{3}$, \\ Priscillia Imelda ${ }^{1}$ (D) \\ ${ }^{1}$ DepartmentofPulmonology, Dharmais National CancerCenter, Jakarta, Indonesia; ${ }^{2}$ DepartmentofMedicalHematology-Oncology, \\ Dharmais National Cancer Center, Jakarta, Indonesia; ${ }^{3}$ Department of Research and Development, Dharmais National Cancer \\ Center, Jakarta, Indonesia
}

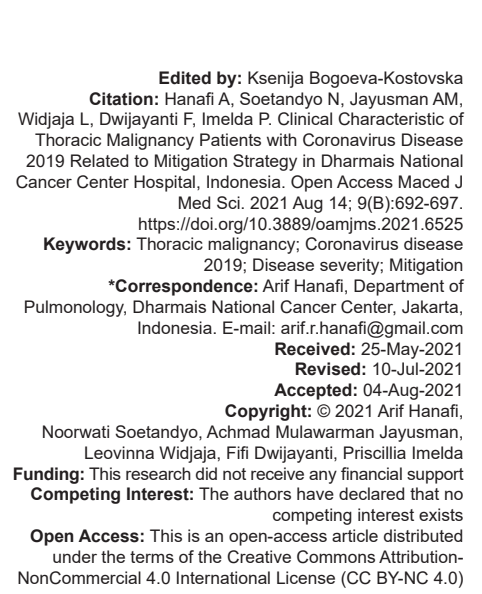

Introduction

Coronavirus disease 2019 (COVID-19), an infection caused by severe acute respiratory syndrome coronavirus 2, has become a significant burden worldwide. The first case was identified in Wuhan, Hubei Province, the People's Republic of China, in December 2019 [1] in which it spreads worldwide rapidly. In March 2020, the World Health Organization (WHO) designated this disease as a pandemic [2]. The pandemic is yet to end after a year has passed. Approximately 120 million cases have been recorded globally from the beginning up to March 16, 2021 [3]. The pandemic has affected almost every aspect of life, including socioeconomic, psychology, and health care. Strict regulations must be made to contain the transmission. This can be in the form of area locked down, limitation of services, and delaying non-urgent complaints.

Patients with cancer are among the most vulnerable people to severe infection. Cancer is independently associated with mortality in patients with COVID-19 [4]. Thus, COVID-19 infection can worsen the condition. Among thoracic malignancy and other cancer, the study showed that lung cancer is the most prevalent type in patients with COVID-19. It is associated with the highest infection rate and deaths caused by COVID-19 and structural damage caused by lung cancer itself, though it could contribute to poor prognosis [5], [6], [7], [8].

Cancer patients often need continuous medical treatment requiring hospitalization. Unfortunately, regulations imposed during the pandemic restrict activities outside the home. Hence, it may cause a delay in cancer treatment which potentially leads to disease progression.

As a developing country facing multifaceted problems in multiple sectors, Indonesia has tried to balance disease containment and economy. Thus, the only partial lockdown was attempted, and the government focused the resources on improving disease-preventing measures and regulations that are 
not related to lockdown. As National Referral Hospital for the cancer patient, Dharmais Cancer Hospital has implemented some health protocols in responding to the pandemic while the health services are still undergoing to avoid treatment delay. Almost all cancer patients who come to our hospital are advanced-stage cancer patient, where 3-6 months survival rate is very low generally. Postponing the treatment might be worsen the condition. Therefore, we applied strict mitigation strategies, although COVID-19 infection still occurs sometimes. In this paper, we present data related to COVID-19 infection in patients with thoracic malignancy. We described the clinical data and laboratory findings that compared to disease severity, to investigate whether COVID-19 progression on cancer patients could be prevented with strict regulation.

\section{Materials and Methods}

\section{Study design and participants}

This was a descriptive study conducted at the Dharmais Cancer Hospital, Jakarta, the National Cancer Center of Indonesia. Data of cancer patients with COVID-19 were obtained from the medical record with research ethical permission. Diagnosis of COVID-19 was made based on the WHO guideline, which was laboratoryconfirmed COVID-19. Reverse transcription polymerase chain reaction (RT-PCR) analysis of samples collected from a nasopharyngeal swab [9].

From May 1, 2020, to March 31, 2021, we enrolled 681 cancer patients infected by COVID-19. About 56 patients were diagnosed with thoracic malignancy based on pathological and radiology examination. We excluded 11 patients because of metastatic lesion origin from other cancer organ and selected 45 primary thoracic malignancy patients then defined the clinical characteristic and disease severity affected by COVID-19 (Figure 1). All these patients were diagnosed with advanced-stage cancer. The patients included all cases of newly diagnosed, remission, progression, or recurrent. This study has approved by the Research Ethics Commission of the Dharmais Cancer Hospital and the need for informed consent was waived due to pandemic.

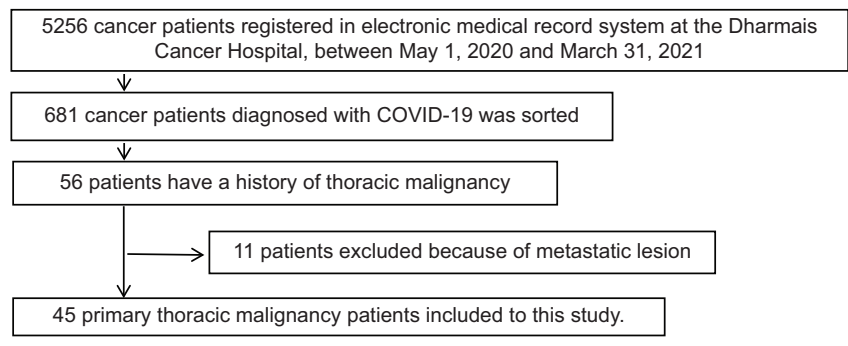

Figure 1: Study profile

\section{Data collection}

Patients medical record was obtained both from electronic and written medical records at the Dharmais Cancer Hospital. Collected data included age, gender, body mass index, comorbidity, cancer type, cancer stage, disease severity, the Eastern Cooperative Oncology Group (ECOG) score, admission status, treatment status, laboratory results, and clinical outcomes. COVID-19 disease severity was determined based on the government's guideline [10]. ECOG score was made on the patient $1^{\text {st }}$ time coming to the hospital. The outcomes included death or life at the end of the event.

\section{Statistical analysis}

We did a univariate and bivariate analysis. The data analyzed in SPSS version 25 . We calculated the relevant descriptive statistics for the characteristic of patients (age, gender, body mass index, comorbidity, lung cancer type, lung cancer stage, disease severity, performance status, hospital care, and treatment status) and laboratory findings. Continuous variables described as median (minimum-maximum) or mean (standard deviation), while categorical variables described as frequencies and percentages. The Wilcoxon ranksum test was used to compare continuous data with more than 2 categorical dependent variables, while the Mann-Whitney test with two categorical variables. The significance level was set at $p<0.05$.

\section{Results}

\section{with COVID-19}

The number of thoracic malignancy patient

All cancer patients with COVID-19 were about $12.9 \%$ of total patients registered. There were a total of 45 thoracic malignancy patients $(6 \%)$ with advanced stage obtained from the medical record. Table 1 shows the demographic and clinical characteristic of thoracic malignancies patient diagnosed with COVID-19. The median age was 59 years ( $\min 20$ years, max 77 years) with the male population $(66.7 \%)$ was twice as female (33.3\%). Most patients had a normal body mass index $(66.7 \%)$ and without comorbidity (66.7\%). Diabetes was the most frequent comorbidity (15.6\%), followed by heart disease $(6.7 \%)$, renal disease $(4.4 \%)$, other diseases (tuberculosis and obesity) (4.4\%), and hypertension $(2.2 \%)$. Lung tumor was the most frequent type among other thoracic malignancy $(84.4 \%)$, which dominated by non-small cell lung cancer (NSCLC) $(57.8 \%)$. There were six cases with mediastinal tumor (13.3\%) and one case of pleural tumor which was mesothelioma (2.2\%). Most patients have end-stage cancer (48.9\%). However, 
there was still an undetermined stage (28.9\%), since some COVID-19 patients undergo self-quarantine and some others deteriorated to death at the $1^{\text {st }}$ day contact. About a half of patients were not having any treatment yet (naive) (53.5\%), 13 patients were undergoing treatment $(28.9 \%)$ and 8 patients have completed the treatment $(17.8 \%)$. Nearly half of patients have severe COVID-19 disease (46.7\%), followed by asymptomatic $(31.1 \%)$, mild $(13.3 \%)$, and moderate disease $(8.9 \%)$. Limited activity illustrated by ECOG score which most patients have 1-3 points. Patient with the asymptomatic and mild disease could undergo outpatient care $(44.4 \%)$. Others need an isolation room. Fifteen out of 21 patients with the severe disease need isolation room

Table 1: Baseline characteristics of thoracic malignancy patients with COVID-19

\begin{tabular}{|c|c|}
\hline Characteristic & $n=45(\%)$ \\
\hline Age & $59(20-77)$ \\
\hline \multicolumn{2}{|l|}{ Age range } \\
\hline Child ( $0-14$ years) & 0 \\
\hline Adolescent (15-24 years) & $2(4.4)$ \\
\hline Young adult ( $25-45$ years) & $8(17.8)$ \\
\hline Middle aged ( $46-64$ years) & $20(44.4)$ \\
\hline Elderly ( $\geq 65$ years) & $15(33.3)$ \\
\hline \multicolumn{2}{|l|}{ Gender } \\
\hline Male & $30(66.7)$ \\
\hline Female & $15(33.3)$ \\
\hline \multicolumn{2}{|l|}{ BMI category } \\
\hline Underweight $(<18.50)$ & $9(20.0)$ \\
\hline Normal (18.50-24.99) & $30(66.7)$ \\
\hline Overweight (25-29.99) & $5(11.1)$ \\
\hline Obesity $(\geq 30)$ & $1(2.2)$ \\
\hline \multicolumn{2}{|l|}{ Comorbidity } \\
\hline Without comorbidity & $30(66.7)$ \\
\hline Hypertension & $1(2.2)$ \\
\hline Diabetic & $7(15.6)$ \\
\hline Heart disease & $3(6.7)$ \\
\hline Renal disease & $2(4.4)$ \\
\hline Others & $2(4.4)$ \\
\hline \multicolumn{2}{|l|}{ Cancer types } \\
\hline Lung tumor & $38(84.4)$ \\
\hline Mediastinal tumor & $6(13.3)$ \\
\hline Pleura tumor & $1(2.2)$ \\
\hline Others & 0 \\
\hline \multicolumn{2}{|l|}{ Pathology types } \\
\hline Undetermined & $12(26.7)$ \\
\hline Non-small cell lung carcinoma & $26(57.8)$ \\
\hline Small cell lung carcinoma & $2(4.4)$ \\
\hline Mesothelioma & $1(2.2)$ \\
\hline Thymoma & $2(4.4)$ \\
\hline Germ cell tumors & $2(4.4)$ \\
\hline \multicolumn{2}{|l|}{ Cancer stage } \\
\hline Undetermined & $13(28.9)$ \\
\hline Stage III $(a, b)$ & $10(22.2)$ \\
\hline Stage IV $(a, b)$ & $22(48.9)$ \\
\hline \multicolumn{2}{|l|}{ Treatment status } \\
\hline Naive & $24(53.3)$ \\
\hline Ongoing treatment & $13(28.9)$ \\
\hline Completed/maintenance & $8(17.8)$ \\
\hline \multicolumn{2}{|l|}{ COVID-19 disease severity } \\
\hline Asymptomatic & $14(31.1)$ \\
\hline Mild & $6(13.3)$ \\
\hline Moderate & $4(8.9)$ \\
\hline Severe & $21(46.7)$ \\
\hline \multicolumn{2}{|l|}{ ECOG score } \\
\hline 0 & $5(11.1)$ \\
\hline 1 & $12(26.7)$ \\
\hline 2 & $14(31.1)$ \\
\hline 3 & $11(24.4)$ \\
\hline 4 & $3(6.7)$ \\
\hline \multicolumn{2}{|l|}{ Hospital care } \\
\hline Outpatient & $20(44.4)$ \\
\hline \multicolumn{2}{|l|}{ Inpatient } \\
\hline Isolation ward only & $5(11.1)$ \\
\hline Isolation ward with negative pressure & $15(33.3)$ \\
\hline Emergency department & $3(6.7)$ \\
\hline ICU with negative pressure & $2(4.4)$ \\
\hline \multicolumn{2}{|l|}{ Outcome } \\
\hline Live & $25(55.6)$ \\
\hline Dead & $20(44.4)$ \\
\hline
\end{tabular}

with negative pressure $(33.3 \%)$, three patients were dead on the first contact at an emergency room (6.7\%), and two patients need ICU (4.4\%). Twenty-five patients were survived $(55.6 \%)$.

\section{Laboratory findings}

Comparative analysis for laboratory findings to disease severity among patients is illustrated in Table 2. The mean result of hemoglobin, hematocrit, erythrocytes, platelets, and leukocytes was 11.4, $33.9,4.0,12.7$, and 353.1, respectively. These results have no significant finding. Meanwhile, neutrophilia (mean 78.0) and lymphopenia (13.0) were significant compared to disease severity. It was also supported by the significant result of high neutrophil-lymphocyte ratio (NLR) (13.1; $p=0.0000)$. There was a significant blood urea increasing among patient (31.6; $p=0.04$ ), with normal blood creatinine and eGFR. All liver function indicators were not significant compared to disease severity. Fibrinogen and d-dimer count were high and have significant results compared to disease severity, $p=0.036$ and $p=0.027$, respectively. C-reactive protein (CRP) was also high (56.8), but it was not significant compared to diseases severity.

Table 2: Laboratory findings of thoracic malignancy patients with COVID-19

\begin{tabular}{|c|c|c|c|}
\hline & Normal range & Univariate analysis & $\begin{array}{l}\text { Bivariate analysis } \\
\text { ( } p \text {-value) }\end{array}$ \\
\hline & & mean (SD) & disease severity\# \\
\hline \multicolumn{4}{|l|}{ Hematology profile } \\
\hline Hemoglobin $(\mathrm{g} / \mathrm{dL})$ & $13-18$ & $11.4(2.2)$ & 0.079 \\
\hline Hematocrit (\%) & $40-54$ & $33.9(6.4)$ & 0.191 \\
\hline Erythrocytes ( $\left.10^{6} / \mathrm{uL}\right)$ & $4.60-6.20$ & $4.0(0.6)$ & 0.381 \\
\hline Platelets $\left(10^{3} / \mathrm{uL}\right)$ & $150-440$ & $353.1(157.4)$ & 0.788 \\
\hline Leukocytes ( $\left.10^{3} / \mathrm{uL}\right)$ & $5.0-10.0$ & $12.7(7.2)$ & 0.396 \\
\hline Segmented neutrophils (\%) & $50-70$ & $78.0(12.6)$ & $0.001^{*}$ \\
\hline Lymphocytes (\%) & $20-40$ & $13.0(9.6)$ & $0.000^{*}$ \\
\hline Monocytes (\%) & $2-8$ & $7.4(5.4)$ & 0.221 \\
\hline ANC $\left(10^{3} / \mathrm{uL}\right)$ & $2.50-7.00$ & $10.6(7.3)$ & 0.109 \\
\hline NLR & $0-5.8$ & $13.1(18.4)$ & $0.000^{*}$ \\
\hline \multicolumn{4}{|l|}{ Renal function profile } \\
\hline Blood urea (mg/dL) & $5-20$ & $31.6(19.1)$ & $0.040^{*}$ \\
\hline Blood creatinine $(\mathrm{mg} / \mathrm{dL})$ & $<1.17$ & $0.9(0.4)$ & 0.735 \\
\hline eGFR $\left(\mathrm{ml} / \mathrm{min} / 1.73 \mathrm{~m}^{2}\right)$ & $>60$ & $116.2(67.8)$ & 0.777 \\
\hline \multicolumn{4}{|l|}{ Liver function profile } \\
\hline SGOT (U/L) & $0-38$ & $31.1(27.7)$ & 0.882 \\
\hline SGPT (U/L) & $0-41$ & $19.1(12.0)$ & 0.061 \\
\hline Total protein $(\mathrm{g} / \mathrm{dL})$ & $6.6-8.7$ & $6.7(0.9)$ & 0.180 \\
\hline Albumin (g/dL) & $3.5-5.4$ & $3.2(0.7)$ & 0.093 \\
\hline Globulin (g/dL) & $2.0-3.9$ & $3.5(1.1)$ & 0.638 \\
\hline \multicolumn{4}{|l|}{ Coagulation factor } \\
\hline PT (second) & $10-12$ & $16.9(12.9)$ & 0.062 \\
\hline aPTT (second) & $20-35$ & $31.4(7.1)$ & 0.941 \\
\hline Fibrinogen (mg/dL) & $187-451$ & $521.7(182.0)$ & $0.036^{*}$ \\
\hline \multicolumn{4}{|l|}{ Inflammatory factors } \\
\hline D-dimer (ng/mL) & $<500$ & $3821.6(6539.3)$ & $0.027^{*}$ \\
\hline $\mathrm{CRP}(\mathrm{mg} / \mathrm{L})$ & $<6$ & $56.8(44.7)$ & 0.599 \\
\hline
\end{tabular}
rate: SGOT: Serum glutamic oxaloacetic transaminase: SGPT: Serum glutamic pyruvate transaminase; PTT. Prothrombin time: aPTT: Activated partial thromboplastin time; CRP: C-reactive protein $(\#)$ : Kruskal-Wallis test, ${ }^{*} p<0.05$

\section{Discussion}

At the beginning of the COVID-19 pandemic, several hospitals in Indonesia were overwhelmed, including Dharmais Cancer Hospital. In general, 
patients with cancer were immunocompromised and vulnerable to infection. However, due to the aggressive nature of cancers, treatment for these patients cannot be postponed. Therefore, Dharmais Cancer Hospital formed the COVID-19 mitigation team. Various efforts have been done, such as double screening, personal protective equipment (PPE) supply, limitation of inpatient visiting hours, construction of isolation room with negative pressure, ICU with negative pressure, and room classification based on colored zone (red, yellow, or green). The purpose was to protect both patient and health care workers at the hospital. At the main entrance of hospital, patients and other visitors would be underwent screening process by temperature checking and form filling by answering short questions related to COVID-19 infection. Every procedure also needs screening approval, including negative result on RT-PCR test. Every room has classified based on the risk of COVID-19 transmission by marking the door with colored zone tag. Red for high-risk zone, yellow for moderate risk, and green one for safe zone. Every health care worker was supplied with PPE. Once patient got positive result on RT-PCR analysis, our mitigation team will trace and contact the patient. Every people who has close relationship and contact with patient would be asked to do nasopharyngeal swab and PCR analysis. Patients with asymptomatic and mild COVID-19 disease were suggested to have self-quarantine at home. This was aimed to reduce the exposure to other vulnerable cancer patients at the hospital. These patients also have their oral medication at home and controlled by the COVID-19 team. Because all these patients have stable condition, postponing the cancer treatment was not a big matter while COVID-19 disease addressed. They would be followed up to 10-14 days after quarantine and do their next examination based on standard protocols until they were proved to have a negative result and allowed to continue cancer treatment at the hospital. Meanwhile, cancer patients with moderate and severe disease should be hospitalized and need advanced treatment, both oral and intravenous medication, where postponing cancer treatment becomes a difficult decision. For patients with moderate COVID19 disease, starting cancer treatment and COVID-19 treatment simultaneously should depend on patient condition and strict monitoring should be made. Severe COVID-19 patients should become a priority and need intensive care isolation unit, while cancer treatment must be considered "the benefit outweigh the risk."

Even though COVID-19 is highly contagious, its transmission can be minimized with correct strategy. It is proved by there was only $12.9 \%$ of cancer patients diagnosed with COVID-19 in our hospital. About $6 \%$ of them with thoracic malignancy reflecting the prevalence of cancers in Indonesia, in which, breast cancer, cervix cancer, and hematologic malignancy are the most common type of cancers [11]. Patients with thoracic malignancies, especially lung cancers, are in dire need of adequate COVID-19 preventive measure due to its vulnerability compared to both the normal population and patients with cancer [5], [6], [7], [8]. Special attention and monitoring should be given to them to prevent disease progression.

The proportion of male patients is significantly higher in patients with thoracic malignancies which may be due to one of the most important risk factors, namely, smoking, which is more frequently observed in male patients, even though the current data did not support the association. The patients mostly have normal weight and no comorbidity. However, several patients have comorbidity, such as diabetic, heart disease, and renal disease, which may affect the disease outcome since comorbidity was known as the risk factor for COVID-19 infection [9], [10]. NSCLC was the most frequent type of thoracic cancer in late-stage cancer (III, IV). Patients with undetermined type and/or stage have varied condition which postponed staging process. Half of them underwent self-quarantine and others deteriorated to death. Several patients experience severe COVID-19, including three patients were brought to the emergency department at the $1^{\text {st }}$ time contact. Nearly half of the patients died. To be precise, 20 patients with severe COVID-19 did not survive.

Several laboratory findings are associated with severe disease and these could be the indicator of poor outcome. Neutrophilia and Iymphopenia, supported by the increasing of NLR, have significant result. The study indicates that in the early phase of COVID-19 infection when inflammation was responded to cytokines storm, all the defender cells could be burst to fight against the infection [12], [13]. Other inflammatory factors, such as d-dimer and CRP, were assessed in this study, but the result is inconclusive. The d-dimer and CRP were increased but CRP was not statistically significant with regard to the disease severity. Several studies have explained that at the early phase of COVID-19 infection, d-dimer and CRP would raise along with COVID19 progression [14], [15]. Thus, the non-significant finding in our study may be due to the small sample size or the increase of d-dimer may not be caused by an inflammatory surge, but coagulation interference, since fibrinogen was also significantly increased.

Our study also significantly showed
hyperuricemia but normal blood creatinine. Theoretically, both urea and creatinine excretion occur in the renal, but urea was synthesized primary from dietary or tissue metabolism, whereas creatinine from muscle catabolism [16]. This basic science strengthened the reason that hyperuricemia in this study may not be caused by kidney injury, instead of tissue metabolism from the inflammatory process. However, our study cannot differentiate whether it was caused by the COVID-19 disease or cancer cell turnover process [16], [17]. A previous study explained that both blood urea and creatinine would 
be elevated, since acute kidney injury may be affected by COVID-19 infection and they both can be used as the predictive factor to the poor outcome [18]. Prothrombin time (PT) was elongated which means that the coagulation cascade may be interrupted, but it is not statistically significant. Compared to the activated partial thromboplastin time (aPTT), PT has the value to explain the external pathway in the coagulation process, while aPTT for the internal pathway. Some factors lead to prolonging PT, one of them was liver disease. Several studies explained that liver injury may occur during COVID-19 infection [19], which may contribute to the prolonged PT. However, our findings did not show altered in liver function, except for albumin which was slightly decreased (hypoalbuminemia) but not significant. On the other hand, albumin was also altered (hypoalbuminemia), but it might be due to cancer itself. Further studies are needed to conclude this hypothesis.

The important point we want to describe that even though cancer patient was vulnerable, and COVID-19 may worsen the condition, it could be addressed with strict mitigation strategies. Cancer treatment along with COVID-19 treatment could be considered to give with strict monitoring. By correct and strict mitigation strategies, we find that only small proportion of COVID-19 disease occurs in cancer patients. Among thoracic malignancy patients, patients with asymptomatic, mild, and moderated, which underwent self-quarantine or hospitalization, were not progress to severe disease. This showed that the strict COVID-19 mitigation strategy in our hospital proves to be successful.

\section{Conclusion}

There is a small proportion of cancer patients with COVID-19. Among thoracic malignancy, most patients do not progress to severe disease, showing that the strict mitigation strategy in our hospital was successful. However, all patients with severe COVID-19 at the first contact did not survive. Laboratory findings, such as neutrophilia, lymphopenia, neutrophil-to-lymphocyte ratio, hyperuricemia, high fibrinogen, and d-dimer, may be valuable for predicting prognosis.

\section{Acknowledgment}

The COVID-19 mitigation team; Department of Research and Development; and Integrated Laboratory of Dharmais National Cancer Center, Jakarta, Indonesia.

\section{Ethical Approval}

This study has approved by Research Ethics Commission of Dharmais Cancer Hospital, with Ethical clearance, No. 149/KEPK/X/2020.

\section{References}

1. Li Q, Guan X, Wu P, Wang X, Zhou L, Tong Y, et al. Early transmission dynamics in Wuhan, China, of novel coronavirusinfected pneumonia. N Engl J Med. 2020;382(13):1199-207.

2. Cucinotta D, Vanelli M. WHO declares COVID-19 a pandemic. Acta Biomed. 2020;91(6):157-60.

PMid:32191675

3. World Health Organization. WHO Coronavirus (COVID-19) Dashboard. Geneva: World Health Organization; 2021.

4. Meng Y, Lu W, Guo E, Liu J, Yang B, Wu P, et al. Cancer history is an independent risk factor for mortality in hospitalized COVID-19 patients: A propensity score-matched analysis. J Hematol Oncol. 2020;13(1):75. https://doi.org/10.1186/ s13045-020-00907-0

PMid:32522278

5. Rogado J, Pangua C, Serrano-Montero G, Obispo B, Marino AM, Pérez-Pérez M, et al. Covid-19 and lung cancer: A greater fatality rate? Lung Cancer. 2020;146(1):19-22. https:// doi.org/10.1016/j.lungcan.2020.05.034 PMid:32505076

6. Dai M, Liu D, Liu M, Zhou F, Li G, Chen Z, et al. Patients with cancer appear more vulnerable to SARS-COV-2: A multi-center study during the COVID-19 outbreak. Cancer Discov. 2020;10(6):78391. https://doi.org/10.1158/2159-8290.cd-20-0422

PMid:32345594

7. Luo J, Rizvi H, Preeshagul IR, Egger JV, Hoyos D, Bandlamudi C, et al. COVID-19 in patients with lung cancer. Ann Oncol. 2020;31(10):1386-96.

PMid:32561401

8. Dai MY, Chen Z, Leng Y, Wu M, Liu Y, Zhou F, et al Patients with lung cancer have high susceptibility of COVID-19: A retrospective study in Wuhan, China. Cancer Control. 2020;27(1):107327482096046. https://doi. org/10.1177/1073274820960467

PMid:32938231

9. World Health Organization. Clinical Management Clinical Management: Living Guidance COVID-19. Geneva: World Health Organization; 2021. Available from: https://www.who.int/ publications/i/item/WHO-2019-nCoV-clinical-2021-1. https://doi. org/10.1053/crad.2001.0896. [Last accessed on $2021 \mathrm{Apr} 17]$.

10. KKBI. Tata Laksana. KKBI Daring. KKBI; 2016; Available from: https://www.kbbi.kemdikbud.go.id/entri/tata laksana. [Last accessed on 2021 Apr 20].

11. World Health Organization. GLOBOCAN. Country-specific Incidence Method, National Country-specific, Mortality. Vol. 858 Geneva: World Health Organization; 2020.

12. Hu B, Huang S, Yin L. The cytokine storm and COVID-19. J Med Virol. 2021;93(1):250-6.

13. Coperchini F, Chiovato L, Croce L, Magri F, Rotondi M. The cytokine storm in COVID-19: An overview of the involvement of the chemokine/chemokine-receptor system. Cytokine Growth Factor Rev. 2020;53(1):25-32. https://doi.org/10.1016/j. 
cytogfr.2020.05.003

PMid:32446778

14. Terpos E, Ntanasis-Stathopoulos I, Elalamy I, Kastritis E, Sergentanis TN, Politou M, et al. Hematological findings and complications of COVID-19. Am J Hematol. 2020;95(7):834-47. https://doi.org/10.1002/ajh.25829

PMid:32282949

15. Ponti G, Maccaferri M, Ruini C, Tomasi A, Ozben T. Biomarkers associated with COVID-19 disease progression. Crit Rev Clin Lab Sci. 2020;57(6):389-99. https://doi.org/10.1080/10408363. 2020.1770685

PMid:32503382
16. Gounden V, Bhatt H, Jialal I. Renal Function Tests. Treasure Island, FL: StatPearls Publishing; 2021.

17. George C, Minter DA. Hyperuricemia. Treasure Island, FL: StatPearls Publishing; 2021.

18. Liu YM, Xie J, Chen MM, Zhang X, Cheng X, Li H, et al. Kidney function indicators predict adverse outcomes of COVID-19. Med (New York, NY). 2021;2(1):38-48.e2.

PMid:33043313

19. Alqahtani SA, Schattenberg JM. Liver injury in COVID-19: The current evidence. United Eur Gastroenterol J. 2020;8(5):509-19. https://doi.org/10.1177/2050640620924157

PMid:32450787 